\title{
Spectral and Non-Linear Analyses of Spontaneous Magnetoencephalographic Activity in Alzheimer's Disease
}

\author{
Jesús Poza, PhD*; Carlos Gómez, PhD; Alejandro Bachiller, MS; and \\ Roberto Hornero, PhD \\ Biomedical Engineering Group, Department "Teoría de la Señal y Comunicaciones e \\ Ingeniería Telemática”, E.T.S. Ingenieros de Telecomunicación, University of \\ Valladolid, Paseo de Belén, 15, 47011 - Valladolid, Spain
}

Submitted April 2011. Accepted for publication December 2011.

\begin{abstract}
Alzheimer's Disease (AD) is considered the most prevalent form of dementia. A definite AD diagnosis is established after examination of brain tissue. However, an accurate identification should be attempted to effectively apply therapeutic strategies. The aim of the present study was to perform regional analysis of spontaneous magnetoencephalographic (MEG) activity to describe brain dynamics in AD. Several spectral and non-linear parameters were calculated to obtain a comprehensive description of the spatial abnormalities in brain dynamics. Our findings showed a significant global slowing of MEG activity in $\mathrm{AD}$, as well as a significant loss of irregularity and complexity in several brain regions. Spectral and non-linear parameters reached classification accuracies of around $80 \%$. The results suggest the potential usefulness of spectral and non-linear parameters to characterize the cognitive and functional abnormalities of dementia. These parameters can yield information useful in clinical AD diagnosis and provide further insights on underlying brain dynamics.
\end{abstract}

Keywords: Alzheimer's disease, magnetoencephalogram, non-linear analysis, spectral analysis

\section{INTRODUCTION}

Alzheimer's disease (AD) is a neurodegenerative pathology characterized by deterioration in cognition and memory, progressive impairment in the ability to carry out activities of the daily living, as well as a number of behavioural and psychological symptoms [1]. AD accounts for about $60 \%$ of all dementia cases in developed and

*Corresponding author: Jesús Poza, Biomedical Engineering Group, Department T.S.C.I.T., E.T.S. Ingenieros de Telecomunicación, University of Valladolid, Paseo de Belén, 15, 47011 - Valladolid, Spain. Phone: +34 983423000 (5569). Fax: +34 983 423667. E-mail: jespoz@tel.uva.es. Other authors: cargom@tel.uva.es; bachillermatarranz@gmail.com; robhor@tel.uva.es. 
developing countries [2, 3], and represents the leading form of age-related dementias. Several risk factors have been identified, with ageing being the most important one [2]. The relevance of ageing is derived from the increase in life expectancy of the world population, which contributes to the AD prevalence $[2,3]$.

Clinical diagnosis of AD is usually based on the criteria of the National Institute of Neurological and Communicative Disorders and Stroke - AD and Related Disorders Association (NINCDS-ADRDA) [4]. The NINCDS-ADRDA guidelines establish AD diagnosis by means of a complete medical history, as well as physical, psychiatric and neurological examinations [5]. Additionally, laboratory studies, such as thyroidfunction tests and assessment of vitamin B12 deficiency, are recommended to identify other forms of dementia and coexisting disorders associated with ageing. Structural neuroimaging techniques such as computer tomography (CT) or magnetic resonance imaging (MRI) are also appropriate to exclude other causes of dementia [5].

Despite the aforementioned test batteries, there are no definitive imaging or laboratory tests to detect AD. Thus, NINCDS-ADRDA criteria establish several categories depending on the confidence in the diagnosis: unlike, possible and probable. A definite diagnosis can only be made by post-mortem examination of brain tissue [5, 6]. Regardless of the difficulties in diagnosis, an accurate and early identification of AD is crucial to significantly alleviate the most devastating symptoms [7], due to the fact that an optimal treatment requires both nonpharmacological and pharmacological interventions in early stages [1]. Advances in diagnosis, treatment and disease management are highly important in coping with AD.

The analysis of electroencephalographic (EEG) and magnetoencephalographic (MEG) recordings is one of the promising tools to help in AD diagnosis. EEG and MEG signals can reflect anatomical and functional deficits of the brain cortex damaged by $\mathrm{AD}$ at an early stage of the disease [8]. Accumulated evidence suggests that the analysis of electromagnetic brain activity during mental rest provides significant insights on the ageing processes and cognitive decline from neurodegeneration [9]. Although both EEG and MEG rhythms are generated by synchronous oscillations of pyramidal neurons, they reflect slightly different characteristics of the electromagnetic brain activity. EEG signals measure the electric fields induced by all primary currents, whereas MEG recordings are sensitive only to current flows oriented parallel to the scalp [10]. Scalp EEG is strongly influenced by several technical and methodological issues and some authors suggested that scalp EEG can be viewed as the result of a spatial filtering of the electrocorticogram by the volume conductor [11]. Therefore, MEG might provide a more accurate vision of the ongoing brain activity than scalp EEG $[10,11]$.

Since 1980s, EEG activity has been widely analyzed to obtain pathological patterns associated with $\mathrm{AD}[9,12,13]$. On the contrary, analysis of MEG patterns associated with $\mathrm{AD}$ is a relatively recent research field $[13,14]$. The abnormalities that $\mathrm{AD}$ produces in spontaneous MEG oscillations were initially analyzed using conventional signal processing methods, such as spectral analysis. Hence, an increase in the relative power of slow frequency bands together with a reduction in the relative power of high frequency bands have been reported in several MEG studies on AD 
[15-21]. The slowing of MEG activity in AD has also been observed by means of spectral quantifiers such as mean frequency [17, 22], individual alpha peak [16, 22, 23], transition frequency [22] and several spectral ratios [20]. On the other hand, several studies have addressed the characterization of non-linear dynamics associated with AD [14]. The fundamental assumption of these studies is that MEG signals are generated by non-linear deterministic processes with non-linear coupling interactions between neuronal populations. Based on the previous idea, a loss of complexity has been described in AD patients' spontaneous MEG activity by means of several nonlinear parameters, such as correlation dimension [24], Lempel-Ziv complexity [25-29], Higuchi's fractal dimension [29, 30] and multiscale entropy [27], although some works have suggested that the loss of complexity could be only found in high frequency bands [31]. Entropy-related statistics have shown that this decrease in complexity is accompanied by a regularity increase. Thus, a global regularity increase has been found using spectral entropies, such as Shannon, Tsallis and Rényi entropies [22, 32, 33]. Non-linear measures, like approximate entropy [29, 32], sample entropy [26, 27, 29] and auto-mutual information [27, 34], support the global loss of irregularity. On the other hand, studies analyzing functional connectivity have identified a decrease of coherence in the alpha [35, 36], beta and gamma bands [35], as well as a general loss of coherence in all frequency bands [15]. Altered temporal correlations [37] and reduced level of synchronization in specific frequency bands $[13,38-40]$ have also been reported, which suggest a loss of functional connectivity. Likewise, it has been shown that AD is characterized by specific changes of long and short distance interactions in several frequency bands [39, 41]. Finally, it is noteworthy that the aforementioned methods can provide complementary information. Thus, an MEG study suggested that the combination of spectral parameters could be useful to improve the classification accuracy in AD detection [22]. Similarly, the combination of spectral and nonlinear parameters has been successfully employed to increase the classification performance of AD patients and controls [32].

The aim of the present study is to perform regional analysis of MEG activity to describe brain dynamics in AD. We analyzed the spontaneous MEG activity in 36 patients with probable $\mathrm{AD}$ and 26 elderly control subjects, using several spectral and non-linear parameters. These methods have shown their ability to characterize the abnormalities associated with AD patients' MEG activity, such as the slowing of the power spectrum, the loss of irregularity from a spectral and a non-linear point of view, and the decrease in complexity. Furthermore, we analyzed and discussed the potential usefulness of these parameters to help in the complex AD diagnosis.

\section{MATERIALS}

\subsection{Subjects}

Thirty-six patients (12 men and 24 women, age $=74 \pm 7 \mathrm{yr}$, mean \pm standard deviation [SD]) from the "Asociación de Familiares de Enfermos de Alzheimer" of Madrid and twenty-six cognitively normal volunteers ( 9 men and 17 women, age $72 \pm 6$ years, mean $\pm \mathrm{SD}$ ) participated in the study. The patients included in the study were men and women 
$>60$ years of age with a diagnosis of probable AD (mild to moderate) according to the guidelines of the NINCDS-ADRDA [4]. They were pseudo-randomly selected and were collaborative in the MEG recording procedure. AD patients were excluded for the following reasons: atypical evolution according to NINCDS-ADRDA criteria (sudden onset, focal neurological findings and convulsions or abnormalities in the first stages of disease evolution); no Mini-Mental State Examination (MMSE) or Global Deterioration Scale/Functional Assessment Staging (GDS/FAST) score; clinical history of stroke, primary psychotic disorder or of any disease that would by itself explain the clinical syndrome; dementia due to multiple aetiologies; history of alcohol or substance abuse; or metal braces or pacemaker that may interfere with the MEG equipment. Their cognitive function was evaluated using the MMSE, whereas functional status was evaluated by means of the GDS/FAST system [42]. AD patients obtained a mean MMSE and GDS/FAST scores of $18.06 \pm 3.36$ and $4.17 \pm 0.45$ (mean \pm SD), respectively. Controls had a mean MMSE and GDS/FAST scores of $28.88 \pm 1.18$ and $1.73 \pm 0.45$ points, respectively. No significant differences were observed in the mean age of both groups $(p$-value $=0.1911>0.05)$. Participants were not taking any medication (cholinesterase inhibitors, benzodiazepines or antidepressives) that could affect the MEG recordings at the time of study. Informed consent was obtained from all controls and all patients' caregivers. The study was approved by the local Research Ethics Committee.

\subsection{MEG Recordings}

Five minutes of spontaneous MEG activity were recorded for each subject using a 148channel whole-head magnetometer (MAGNES $2500 \mathrm{WH}, 4 \mathrm{D}$ Neuroimaging), placed in a magnetically shielded room in the "Centro de Magnetoencefalografía Dr. PérezModrego" of the Complutense University of Madrid. Subjects were asked to stay in a relaxed state, awake and with eyes closed during MEG acquisition. The recording equipment used in this study acquired the MEG signals at $678.17 \mathrm{~Hz}$ and implemented both a hardware band-pass filter between $1 \mathrm{~Hz}$ and $200 \mathrm{~Hz}$, and a $50 \mathrm{~Hz}$ notch filter. The MEG data were then decimated by filtering the recordings with an anti-aliasing filter according to the Nyquist criterion and down-sampling by a factor of 4 to reduce the data length. The resulting sampling frequency was $169.54 \mathrm{~Hz}$. Afterwards, the recordings were copied as ASCII files to a personal computer. Artifact-free epochs of length 5 s (848 samples) were selected for further analysis. Prior to time-frequency analysis, each MEG signal of 848 samples was digitally band-pass filtered with a Hamming window and cut-off frequencies at 0.5 and $40 \mathrm{~Hz}$. According to the sampling theorem, the upper bound for the frequency components in our MEG signals (i.e., the Nyquist frequency) should be $84.77 \mathrm{~Hz}$ in order to avoid aliasing. Hence, it should be noticed that the proposed cut-off frequencies enable to retain the relevant spectral information in the MEG recordings. Figure 1 displays raw MEG epochs of $5 \mathrm{~s}$ from five sensors for an $\mathrm{AD}$ patient (Figure 1.a) and a control subject (Figure 1.b). The sensors correspond to central channels of five brain regions considered for topographic analyses (i.e. central region: 001; anterior region: 050; posterior: 083; left lateral region: 098; right lateral region: 109). 
(a)
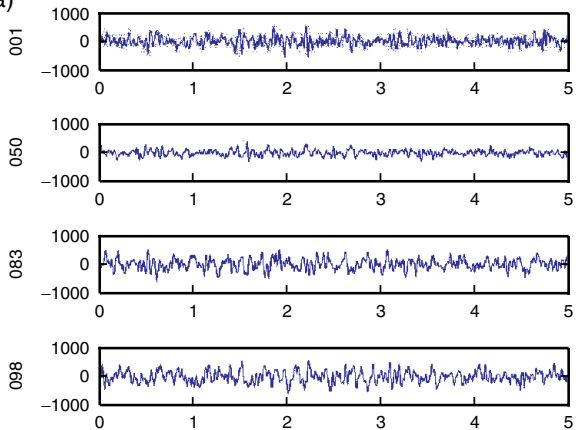

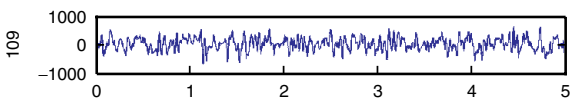

(b)
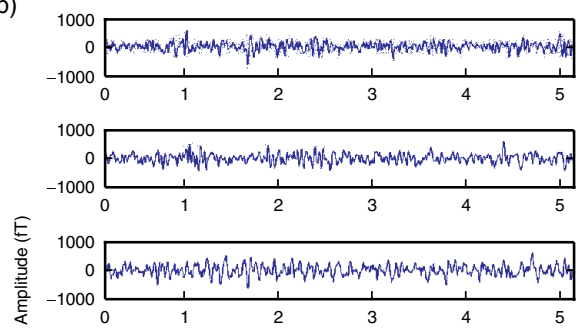

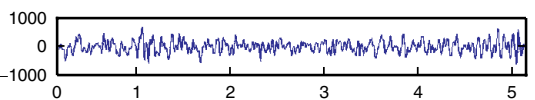

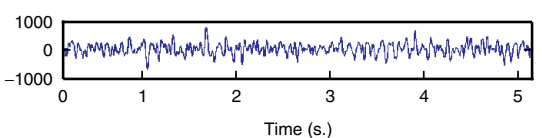

Figure 1. Raw MEG epochs of $5 \mathrm{~s}$ from central channels of five brain regions (i.e., central region: 001; anterior region: 050; posterior: 083; left lateral region: 098; right lateral region: 109). (a) AD patient. (b) Control subject.

\section{METHODS}

\subsection{Spectral Parameters}

A typical approach to characterize electromagnetic brain recordings consists in the analysis of their spectral content. In order to describe the properties of the power spectrum, several spectral parameters have been defined. They are based on the power spectral density (PSD) that represents how the power is distributed in the frequency domain. The Wiener-Khinchin-Einstein theorem states that PSD can be calculated as the Fourier transform of the autocorrelation function, since they are Fourier transforms pairs. In this work, the spectral estimation method provides a frequency resolution of $0.10 \mathrm{~Hz}$. PSD is usually used as a useful and intuitive way to analyze the characteristics of a signal. Likewise, the spectral content between cut-off frequencies $f_{1}$ and $f_{2}$ was selected and PSD was normalized to a scale from 0 to 1 , leading to the normalized PSD $\left(\mathrm{PSD}_{\mathrm{n}}\right)$. It is noteworthy that $f_{1}$ and $f_{2}$ are the cut-off frequencies of the digital band-pass filter 0.5 and $40 \mathrm{~Hz}$, respectively. After the normalization, $\mathrm{PSD}_{\mathrm{n}}$ can be considered as a probability distribution. The following spectral parameters will be defined using this function.

\subsubsection{Relative Power (RP)}

The first parameter that we considered in the spectral analysis was relative power $(R P)$. It represents the relative contribution of several oscillatory components to the global power spectrum. Thus, it is useful to analyze the changes in the spectral content of MEG recordings. Two advantages of relative power compared to absolute power are related to its ability to obtain both independent thresholds from the measurement equipment and lower inter-subject variability. Therefore, it has been recommended in previous EEG studies to 
be employed to analyze dementia [43]. $R P$ is obtained by summing the contribution of the desired spectral composnents. $R P$ was calculated in the conventional EEG frequency bands: delta $(0.5-4 \mathrm{~Hz})$, theta $(4-8 \mathrm{~Hz})$, alpha $(8-13 \mathrm{~Hz})$, beta $1(13-19 \mathrm{~Hz})$, beta 2 $(19-30 \mathrm{~Hz})$ and gamma $(30-40 \mathrm{~Hz})$. It is noteworthy that frequency boundaries were set taking into account that MEG recordings were filtered between 0.5 and $40 \mathrm{~Hz}$.

\subsubsection{Median Frequency $(M F)$}

An alternative way to quantify the changes in the spectral content of MEG recordings is to calculate several characteristic frequencies that describe the behaviour of the most relevant oscillatory components. The median frequency $(M F)$ offers a simple way of summarizing the whole spectral content of PSD. It is defined as the frequency which comprises $50 \%$ of the power. A parameter strongly related to $M F$ is the mean frequency whose original definition is based on the computation of the spectral centroid. However, previous MEG studies have shown that $M F$ provides a better performance to characterize the spectral changes associated with dementia than the mean frequency [22].

\subsubsection{Individual Alpha Frequency (IAF)}

The individual alpha frequency $(I A F)$, also named peak frequency, quantifies the frequency at which the maximum alpha power is reached. Alpha oscillations are dominant in the EEG of resting normal subjects, with the exception of irregular activity in the delta band and lower frequencies [44]. This issue involves that PSD displays a peak around the alpha band. The estimation of $I A F$ in the present work was based on the calculation of the median frequency in the extended alpha band $(4-15 \mathrm{~Hz})$, as previous MEG studies on dementia have recommended [22]. Thus, problems related to the appearance of several peaks in the considered frequency range or spurious spectral components can be avoided.

\subsubsection{Spectral Entropy (SE)}

Entropy is a thermodynamic function, which was adapted to the context of information theory. Its original definition implies uncertainty of information in terms of disorder, discrepancy and diversity. Powell and Percival introduced in 1979 an entropy definition where the irregularity in the signal was estimated in terms of the flatness of the power spectrum [45]. Thus, Shannon's entropy $(S E)$ can be considered as a disorder quantifier [45]. A uniform power spectrum with a broad spectral content (e.g., a highly irregular signal like white noise) provides a high entropy value. On the contrary, a narrow power spectrum with only a few spectral components (e.g., a highly predictable signal like a sum of sinusoids) yields a low entropy value. In the present work, the definition of $S E$ is based on Shannon's entropy computed over the normalized power spectrum. Moreover, $S E$ was normalized to scale from 0 to 1.

\subsection{Non-Linear Parameters}

In order to complement the results provided by the spectral analyses, several non-linear methods were also applied. Increasing evidence suggests that ongoing oscillations in the brain may be weakly non-linear, which appears evident in the case of the alpha 
rhythm [13]. Thus, non-linear methods have opened up a range of new perspectives for the study of normal and disturbed brain function [13, 27]. There is a wide variety of non-linear parameters. Traditionally used non-linear parameters, such as correlation dimension $\left(D_{2}\right)$ and largest Lyapunov exponent $(L 1)$, require noise-free stationary data [46]. These theoretical limitations of $D_{2}$ and $L 1$ involve that the characterization of spontaneous activity should be addressed using other nonlinear techniques suitable for noisy, non-stationary and high-dimensional data [27]. Due to this issue, in the present work, we selected the following non-linear parameters.

\subsubsection{Sample Entropy (SampEn)}

Sample entropy (SampEn) is an embedding entropy, which can be applied to short and relatively noisy time series [47]. SampEn was proposed by Richman and Moorman as an alternative to quantify the irregularity and reduce the bias introduced in the approximate entropy (ApEn) algorithm [47]. Thus, SampEn is largely independent of the sequence length and shows relative consistency under circumstances where ApEn does not [47]. SampEn is also a family of statistics, where two input parameters should be specified: a run length $m$ and a tolerance window $r$. SampEn is the negative natural logarithm of the conditional probability that two sequences similar for $m$ points remain similar at the next point [47]. In this work, SampEn was calculated with parameter values $m=1$ and $r=0.25$ times the SD of the original data sequence. Further details about the SampEn algorithm can be found in [47].

\subsubsection{Lempel-Ziv Complexity (LZC)}

In order to complement the description provided by the embedding entropy, two complexity measures were also used. The first one is Lempel-Ziv complexity $(L Z C)$, a non-parametric measure that evaluates the randomness of finite sequences, which could be also interpreted as a harmonic variability metric [48]. Complex data lead to high $L Z C$ values and regular sequences provide low $L Z C$ values. It is noteworthy that the signal must be coarse-grained, since the $L Z C$ algorithm analyzes a finite symbol sequence [49]. A binary (zeros and ones) conversion was used, as previous studies found that such conversion may retain enough signal information [49]. The detailed algorithm for the measure of $L Z C$ can be found in $[49,50]$.

\subsubsection{Higuchi's Fractal Dimension (HFD)}

Another nonlinear parameter useful to quantify the complexity of a given signal is fractal dimension $(F D)$. The concept of $F D$ was introduced by Mandelbrot to study temporal or spatial continuous phenomena that show correlation to a range of scales [51]. It can be interpreted as a quantifier of the complexity and self-similarity of a sequence [52]. Although many algorithms have been proposed to compute $F D$, such as those introduced by Maragos and Sun [53] or Katz [54], the Higuchi's fractal dimension $(H F D)$ presents several advantages. $H F D$ provides a more accurate estimation of the $F D$ than those by other methods. In addition, $H F D$ is simpler and faster than classical measures derived from chaos theory, like $D_{2}$ and $L 1$, since $F D$ is calculated directly from the time series. HFD is also more sensitive to the noise level than other measures, 
leading to a sensible, slightly distorted translation towards higher $F D$ values [52]. The detailed algorithm for the computation of $H F D$ can be found in [55].

\subsection{Statistical Analysis}

Initially, a descriptive analysis was performed to analyze the characteristics of the dataset. However, variables did not meet homoscedasticity assumption. Therefore, Welch $t$-test was used for the statistical comparison between AD patients and control subjects. It is noteworthy that the statistical analysis was divided into two steps: (i) a descriptive statistical analysis to obtain a graphical description of the spatial distribution of significant differences, and (ii) a regional statistical analysis to accurately analyze the spatial patterns of statistical differences. In step (i), a descriptive statistical analysis was carried out to explore spatial differences between both populations, applying Welch $t$-test to each sensor. In the spectral analysis, the $\mathrm{PSD}_{\mathrm{n}}$ was computed for each $5 \mathrm{~s}$ artifact-free MEG segment and results were averaged for each sensor. Spectral parameters were computed from this function to obtain a value per sensor and subject. Likewise, nonlinear parameters were computed for each artifact-free epoch within the five-minute period of recording and averaged to obtain a value for each sensor and subject. In step (ii), spectral and non-linear parameters were averaged in five regions (anterior, central, left lateral, posterior and right lateral) and analyzed using Welch $t$-test with Bonferroni's correction. Figure 2 depicts the sensor-grouping [33,40] which is included as default sensor groups in the 4D-Neuroimaging source analysis software.

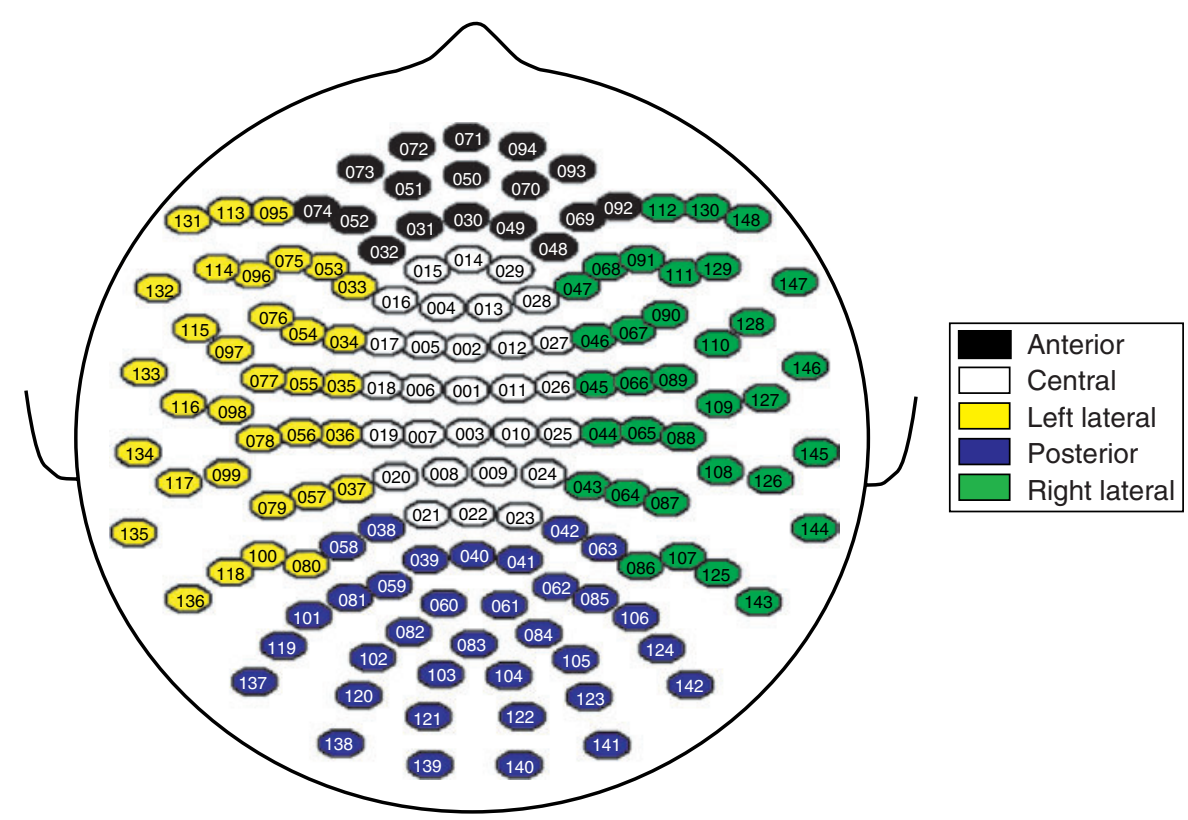

Figure 2. Representation of sensor-grouping into five regions considered for topographic analyses: anterior, central, left lateral, posterior and right lateral. 
The ability of the parameters to distinguish between the two groups was assessed by means of receiver operating characteristic (ROC) curves with a leave-one-out crossvalidation procedure (LOO-CV). It is noteworthy that spectral and non-linear parameters were averaged for each region (anterior, central, left lateral, posterior and right lateral) to obtain a quantitative measure per subject and region. The averaged values were used as inputs to the ROC analysis. Optimum cut-off values were calculated for each ROC model in order to maximize the accuracy. Thus, they correspond to the point closest to the upper left corner of the ROC curve. The area under ROC curve (AUC) was employed as a global measure to summarize the diagnostic ability of each method. In addition, parameters whose AUC was higher than 0.780 were further analyzed in terms of sensitivity (i.e., percentage of patients with a correct AD diagnosis), specificity (i.e., proportion of healthy subjects properly detected) and accuracy (i.e., total fraction of AD patients and healthy subjects well classified).

Both statistical and classification analyses were performed using Matlab ${ }^{\circledR}$ (version 7.0; Mathworks, Natick, MA).

\section{RESULTS}

\subsection{Spectral Parameters}

First, the spectral content of AD patients' MEG activity was assessed using the relative power in the conventional frequency bands. Relative power values reflected a general increase in low frequency bands (delta and theta) and a general decrease in high frequency bands (beta1, beta2 and gamma). Detailed results for relative power values at each frequency band are shown in Figure 3, where the differences in the spatial distributions can be observed. Specifically, the analyses showed a significantly higher $R P$ (delta) (central, left lateral and right lateral regions, $p<0.05$; anterior region, $p<$ 0.01 ) and $R P$ (theta) (central, left lateral and posterior regions, $p<0.01$; right lateral region, $p<0.001)$ in $\mathrm{AD}$ patients than controls. Also in $\mathrm{AD}$ patients, some lower tendencies were found in $R P($ alpha), though statistically significant only in the anterior region $(p<0.05)$. Significantly lower values over all brain regions in AD patients were found with $R P($ beta1) and $R P($ beta2) $(p<0.05$; right lateral region, $p<0.001)$, whereas $R P$ (gamma) exhibited some higher tendencies (anterior, left lateral and right lateral regions, $p<0.05)$.

The "shift-to-the-left" of the power spectrum was also analyzed by means of two additional spectral parameters: $M F$ and $I A F$. Both parameters summarize the distribution of the PSD function, though the $M F$ provides a global summary and the IAF is related to the dominant frequency of alpha frequency band. The distribution of the $M F$ for each group and the corresponding $p$-values are presented in Figure 4, where AD patients exhibited significantly lower $M F$ values than controls over all brain regions (central region, $p<0.01$; anterior, left lateral, right lateral and posterior regions, $p<$ $0.001)$. For $I A F$, Figure 5 depicts the distribution of the parameter for both groups and the corresponding $p$-values. Similar to $M F$, AD patients showed significantly lower IAF values than controls over all brain regions (anterior and posterior regions, $p<0.01$; central, left lateral and right lateral regions, $p<0.001$ ), though the statistical analyses with $I A F$ demonstrated lower $p$-values than those with $M F$. 
(a)

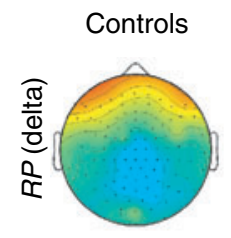

(b)

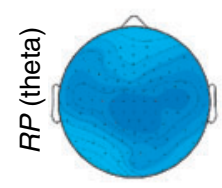

(c)

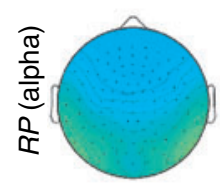

(d)

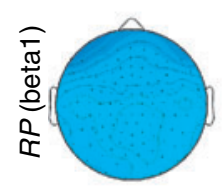

(e)

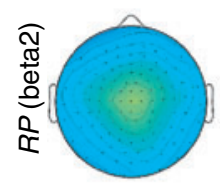

(f)

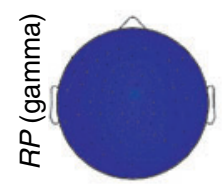

AD patients
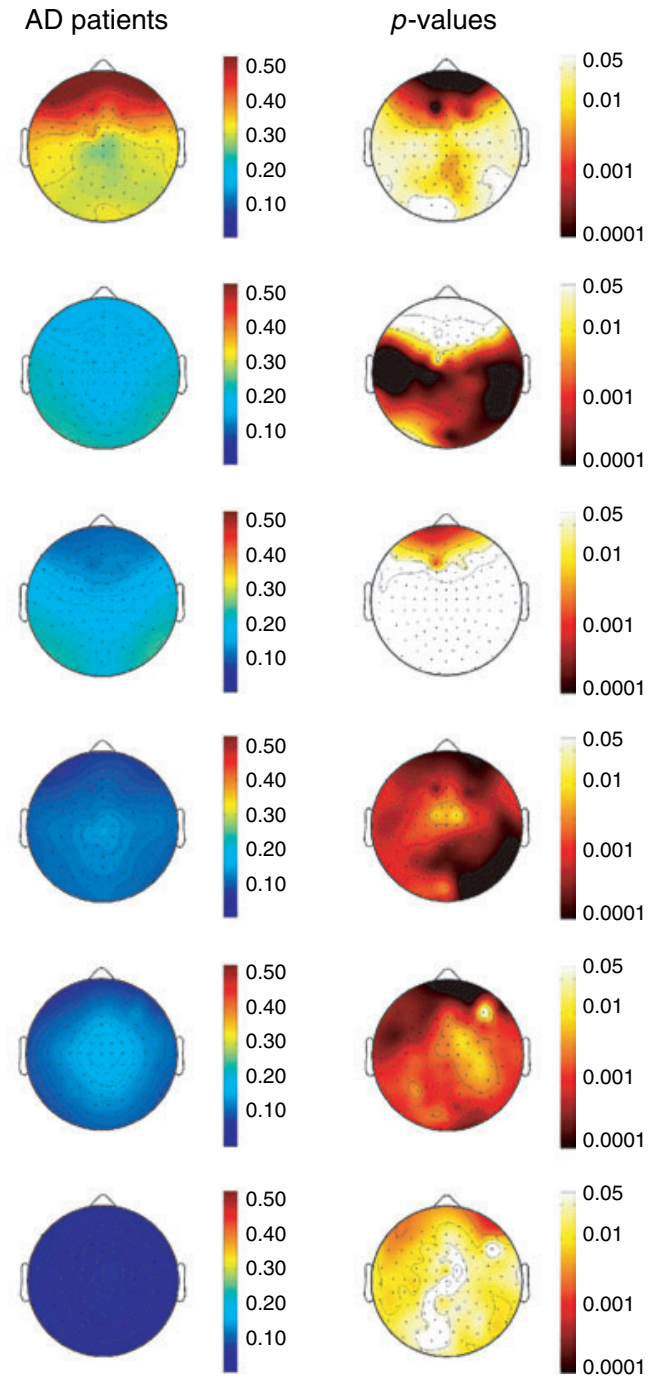

Figure 3. Spatial distribution of the mean relative power values at different frequency bands and the significant differences between the two groups: (a) $R P$ (delta), (b) $R P$ (theta), (c) $R P($ alpha), (d) $R P$ (beta1), (e) $R P$ (beta2), (f) $R P$ (gamma).

The $S E$ quantifies the irregularity of MEG activity in terms of the flatness of the power spectrum. The distribution of the $S E$ for each group, together with the results of the spatial statistical analysis, is displayed in Figure 6. AD patients demonstrated significantly lower $S E$ values than controls in anterior, central and right lateral regions 

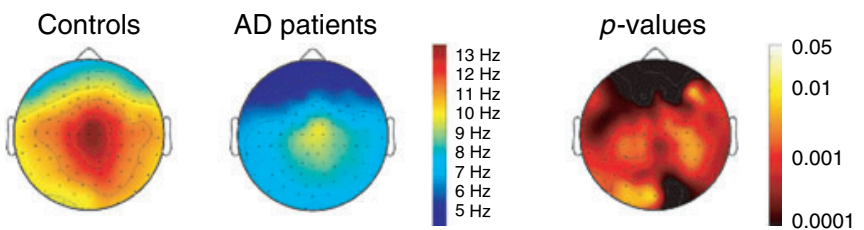

Figure 4. Spatial distribution of $M F$ and its significant difference between the two groups.
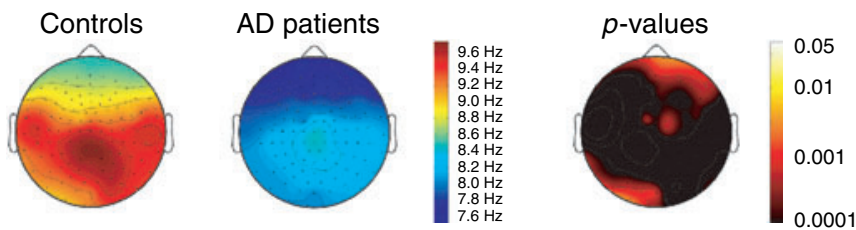

Figure 5. Spatial distribution of $I A F$ and its significant difference between the two groups.
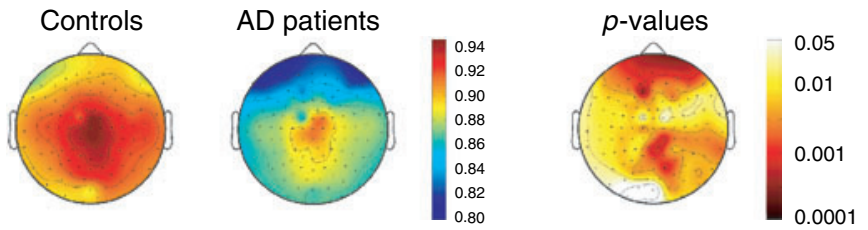

Figure 6. Spatial distribution of $S E$ and its significant difference between the two groups.

$(p<0.01)$. Such results suggest a significant lower irregularity in AD patients' MEG activity in terms of the flatness of the power spectrum.

Finally, ROC curves with LOO-CV were generated to assess the performance of the spectral parameters in distinguishing between AD patients and control subjects. ROC curves for relative power values and the other spectral parameters $(M F, I A F$ and $S E)$ in all brain regions are displayed in Figures 7 and 8 , respectively. In addition, classifications statistics using ROC curves with LOO-CV for parameters with AUC higher than 0.780 are summarized in Table 1 . The highest accuracy was achieved by $S E$ in the anterior region (82.3\%, accuracy; $86.1 \%$, sensitivity; $76.9 \%$, specificity; 0.791 , AUC), whereas the highest AUC was reached by $S E$ in the right lateral region $(75.8 \%$, accuracy; $88.9 \%$, sensitivity; $57.7 \%$, specificity; 0.806, AUC). It is noteworthy that slightly lower accuracies and similar AUC values to those of $S E$ were obtained by $R P($ delta $)$ in the anterior region $(80.7 \%$, accuracy; $86.1 \%$, sensitivity; $73.1 \%$, specificity; 0.788, AUC) and IAF in the central region $(80.7 \%$, accuracy; $75.0 \%$, sensitivity; $88.5 \%$, specificity; 0.803, AUC). 


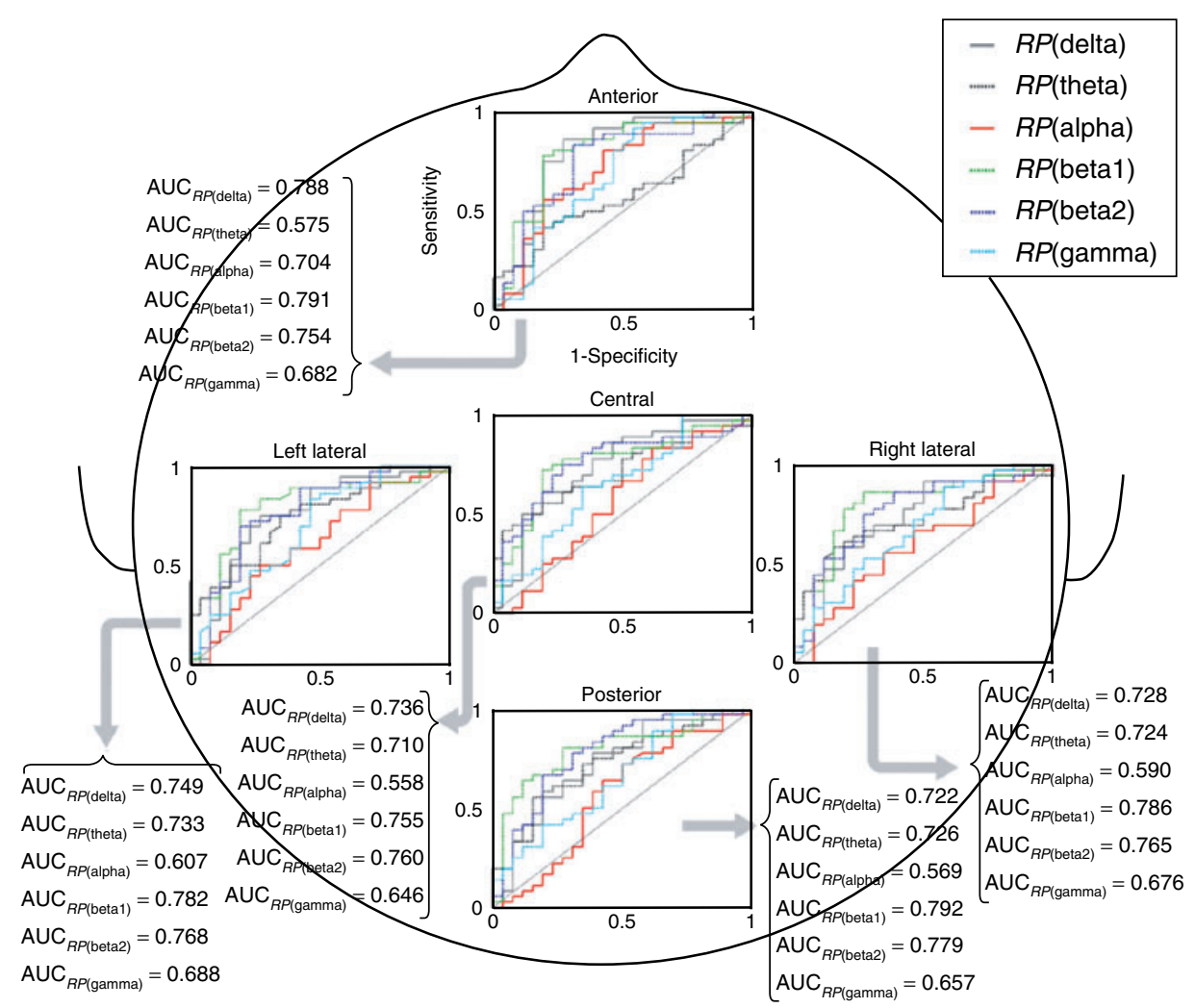

Figure 7. ROC curves showing the performance of relative power, $R P($ delta), $R P$ (theta), $R P$ (alpha), $R P$ (beta1), $R P$ (beta 2) and $R P$ (gamma), in discriminating between $\mathrm{AD}$ patients and controls, and the corresponding AUC values for all brain regions (anterior, central, left lateral, posterior and right lateral).

\subsection{Non-Linear Parameters}

First, SampEn was calculated to measure the irregularity of the MEG activity. SampEn provides information about the fluctuations with time of the MEG signal, comparing the time series with a delayed version of itself [56]. Figure 9 depicts the distribution of SampEn for each group with the corresponding $p$-values. The analyses showed lower SampEn over all brain regions in AD patients than controls, suggesting that $\mathrm{AD}$ is accompanied with a higher MEG regularity. However, significant difference was only observed in the central region $(p<0.05)$.

In order to complement the results obtained by SampEn, two additional non-linear parameters were defined to quantify the complexity of MEG recordings. The first one is $L Z C$. As mentioned previously, a binary conversion was performed for the 


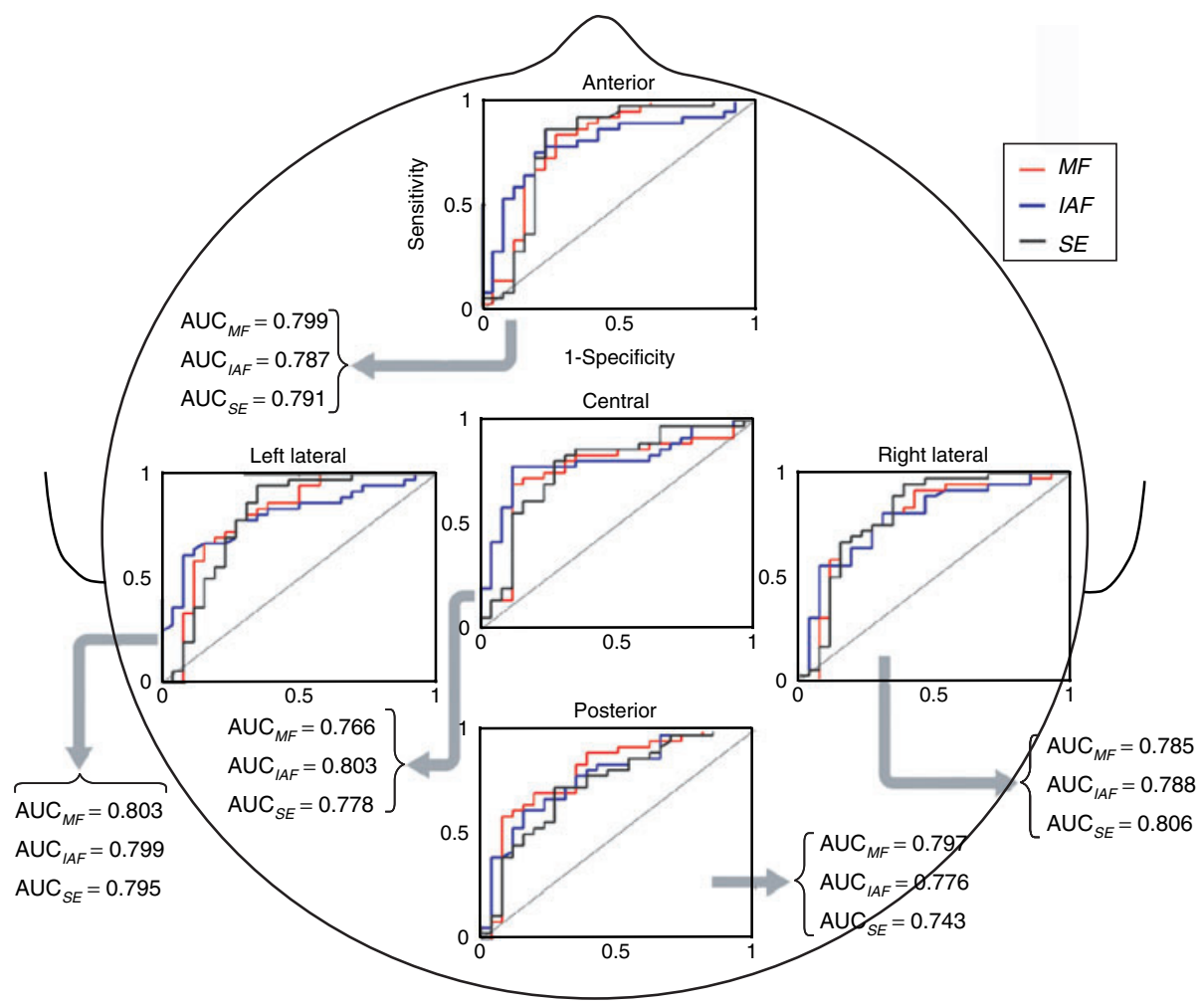

Figure 8. ROC curves showing the performances of $M F, I A F$ and $S E$ in discriminating between $\mathrm{AD}$ patients and controls, and the corresponding AUC values for all brain regions (anterior, central, left lateral, posterior and right lateral).

coarse-grained process and $20 \mathrm{~s}$ artifact-free epochs (3392 samples) were employed following the result of a previous study that $L Z C$ values become stable for MEG signals longer than 3000 samples [25]. Figure 10 presents the distributions of $L Z C$ values for both groups, as well as the corresponding $p$-values. The distributions of $L Z C$ values and significant difference followed a similar pattern as SampEn. Thus, AD patients demonstrated significant lower $L Z C$ values than controls over all brain regions (left lateral, right lateral and posterior regions, $p<0.05$; anterior and central regions, $p<0.01$ ), suggesting that $\mathrm{AD}$ is associated with a lower complexity of MEG activity.

The non-linear parameter HFD was calculated to provide an additional description of the complexity patterns of MEG recordings. It is noteworthy that an accurate estimation of $H F D$ requires stationary MEG epochs. Thus, we applied the Bendat and Piersol's runs test [57] to assess the stationarity of the $5 \mathrm{~s}$ artifact-free epochs. We found that $57.41 \%$ of the epochs were weak or wide sense stationary. These epochs were then selected to compute HFD. The distributions of HFD for both groups and the corresponding $p$-values 
Table 1. Classification statistics (sensitivity, specificity, accuracy and AUC) for the spectral parameters with AUC higher than 0.780

\begin{tabular}{|c|c|c|c|c|c|}
\hline Parameter & Region & Sensitivity $(\%)$ & Specificity $(\%)$ & Accuracy (\%) & AUC \\
\hline$R P($ delta $)$ & Anterior & 86.1 & 73.1 & 80.7 & 0.788 \\
\hline$R P($ beta 1$)$ & Anterior & 75.0 & 76.9 & 75.8 & 0.791 \\
\hline$R P($ beta 1$)$ & Left lateral & 77.8 & 73.1 & 75.8 & 0.782 \\
\hline$R P($ beta 1$)$ & Posterior & 80.6 & 73.1 & 77.4 & 0.792 \\
\hline$R P($ beta 1$)$ & Right lateral & 77.8 & 69.2 & 74.2 & 0.786 \\
\hline$M F$ & Anterior & 80.6 & 69.2 & 75.8 & 0.799 \\
\hline$M F$ & Left lateral & 66.7 & 42.3 & 56.5 & 0.803 \\
\hline$M F$ & Posterior & 83.3 & 61.5 & 74.2 & 0.797 \\
\hline$M F$ & Right lateral & 80.6 & 57.7 & 71.0 & 0.785 \\
\hline$I A F$ & Anterior & 72.2 & 76.9 & 74.2 & 0.787 \\
\hline$I A F$ & Central & 75.0 & 88.5 & 80.7 & 0.803 \\
\hline$I A F$ & Left lateral & 66.7 & 73.1 & 69.4 & 0.799 \\
\hline$I A F$ & Right lateral & 75.0 & 69.2 & 72.6 & 0.788 \\
\hline$S E$ & Anterior & 86.1 & 76.9 & 82.3 & 0.791 \\
\hline$S E$ & Left lateral & 91.7 & 61.5 & 79.0 & 0.795 \\
\hline \multirow[t]{2}{*}{$S E$} & Right lateral & 88.9 & 57.7 & 75.8 & 0.806 \\
\hline & Controls & AD patients & $\begin{array}{l}1.3 \mathrm{~Hz} \\
1.2 \mathrm{~Hz} \\
1.1 \mathrm{~Hz} \\
1.0 \mathrm{~Hz} \\
0.9 \mathrm{~Hz} \\
0.8 \mathrm{~Hz}\end{array}$ & $\begin{array}{l}0.05 \\
0.01 \\
0.001\end{array}$ & \\
\hline
\end{tabular}

Figure 9. Spatial distribution of SampEn and its significant difference between the two groups.
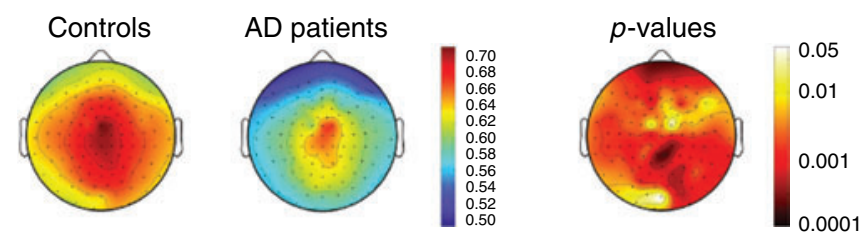

Figure 10. Spatial distribution of $L Z C$ and its significant difference between the two groups.

in Figure 11 show that $\mathrm{AD}$ patients exhibit significant lower $H F D$ values than controls over all brain regions except the left lateral region where only some decreasing tendencies were observed (right lateral and posterior, $p<0.05$; anterior and central regions, $p<0.01$ ). 

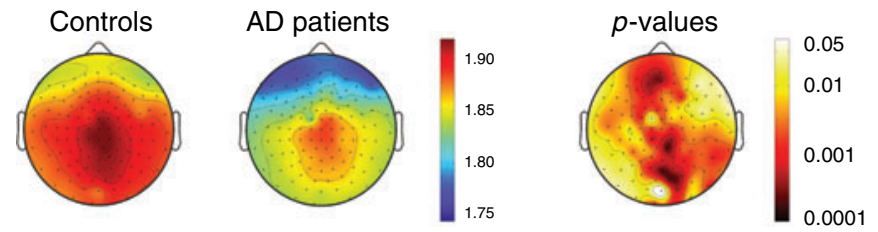

Figure 11. Spatial distribution of $H F D$ and its significant difference between the two groups.

ROC curves for the non-linear parameters (SampEn, LZC and HFD) in all brain regions are displayed in Figure 12. In addition, classifications statistics using ROC curves with LOO-CV for parameters with AUC higher than 0.780 are shown in Table 2. The highest accuracy was achieved by LZC in the left lateral region (79.0\%, accuracy;

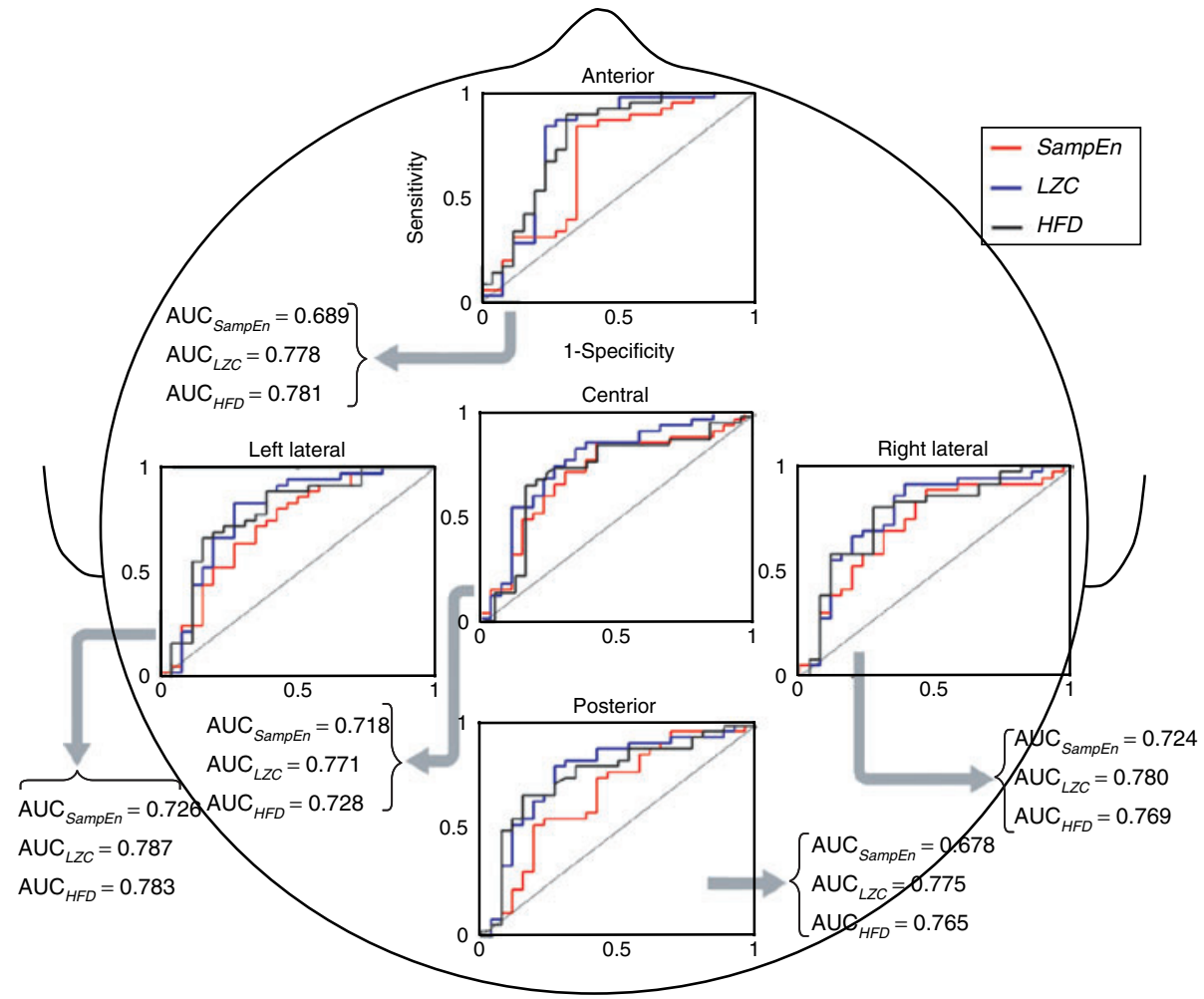

Figure 12. ROC curves showing the performances of SampEn, LZC and HFD in discriminating between AD patients and controls, and the corresponding AUC values for all brain regions (anterior, central, left lateral, posterior and right lateral). 
Table 2. Classification statistics (sensitivity, specificity, accuracy and AUC) for the non-linear parameters with AUC higher than $\mathbf{0 . 7 8 0}$

\begin{tabular}{lccccc}
\hline Parameter & Region & Sensitivity $(\%)$ & Specificity $(\%)$ & Accuracy (\%) & AUC \\
\hline LZC & Left lateral & 83.3 & 73.1 & 79.0 & 0.787 \\
LZC & Right lateral & 86.1 & 61.5 & 75.8 & 0.780 \\
$H F D$ & Anterior & 86.1 & 65.4 & 77.4 & 0.781 \\
$H F D$ & Left lateral & 88.9 & 57.7 & 75.8 & 0.783
\end{tabular}

$83.3 \%$, sensitivity; $73.1 \%$, specificity; 0.787 , AUC), though it was still lower than that of $S E$ in the anterior region. Moreover, lower classification statistics were achieved by $H F D$ in the anterior region $(77.4 \%$, accuracy; $86.1 \%$, sensitivity; $65.4 \%$, specificity; 0.781, AUC) in comparison to $L Z C$.

\section{DISCUSSION}

In this work, the performance of several spectral and non-linear parameters in characterizing spontaneous MEG activity of 36 AD patients and 26 healthy subjects was tested. Our findings suggest that AD affects spontaneous MEG activity by increasing slow rhythms and decreasing fast oscillations. On the other hand, statistically significant decreases were observed in spectral and non-linear entropies in AD patients, suggesting that $\mathrm{AD}$ is associated with an increased regularity. Likewise, complexity quantifiers are significantly lower in $\mathrm{AD}$ patients than controls, suggesting that $\mathrm{AD}$ causes a loss of complexity in spontaneous MEG rhythms. ROC analysis showed classification statistics with accuracies and AUCs of around $80 \%$ and 0.800 , respectively.

Further inspection of relative power values reveals that AD patients reached significantly higher $R P$ values in low frequency bands and significantly lower $R P$ values in high frequency bands than controls. These findings suggest that $A D$ is associated with a slowing of spontaneous MEG activity, in agreement with results from previous investigations $[15,17,18,20]$. The significant "shift-to-the-left" of the power spectrum in $\mathrm{AD}$ was also observed by means of $M F$ and $I A F$. These spectral parameters are significantly lower in AD patients than controls, in agreement with previous studies $[16,22]$. Spatial patterns of significant differences also agree with those reported in previous MEG studies, where AD patients exhibited higher absolute low frequency with a fronto-central maximum and a lower high frequency power values over the occipital and temporal areas in comparison with healthy controls [15].

Similar spatial distributions of abnormalities were obtained by previous MEG studies performing source analysis. Fernández et al. [58, 59] reported a significant increase in the dipole density of slow-wave activity in temporo-parietal regions, which agrees with the results of Osipova et al. that the strongest activation in theta band in the right temporal region [23] and parieto-occipital deficits [16] was observed in AD patients. The results of Osipova et al. [23] support the hemispheric asymmetry in the 
spatial patterns of significant differences that we observed with $R P$ (theta), $R P$ (beta1) and $R P$ (beta2) in $\mathrm{AD}$ patients. Specifically, our findings suggest that the right lateral region is slightly more affected by dementia than the left lateral one. Some investigations indicate that the predominance of $\mathrm{AD}$ in the right hemisphere is associated with a significant impairment in cognitive function [60]. It is noted that our $\mathrm{AD}$ patients were of moderate to severe degree of dementia. Therefore, a right hemispheric predominance in the patterns of significant differences is expected. The lack of significant differences in the alpha band in the posterior areas can be also related to the moderate to severe cognitive impairment of the participating AD patients. Similar results were also reported in previous MEG studies where the alpha band did not show significant differences $[18,20]$. Likewise, the significant changes of $R P($ alpha) in the anterior area is consistent with single dipole source analyses of EEG data, which suggest that alpha and beta activities in the anterior area are affected as a function of AD severity [61].

There is long standing evidence suggesting that the cholinergic deficit plays a critical role in the EEG slowing associated with AD [12]. Analogously, some findings support that MEG slowing in $\mathrm{AD}$ could also be due to the loss of neurotransmitter acetylcholine, since the cholinergic system modulates spontaneous cortical activity at theta and alpha bands and functional coupling in the theta band [62]. Source analyses of MEG signals revealed that $\mathrm{AD}$ is accompanied with a higher dipole density at delta and theta bands $[58,59]$, which suggests that MEG slowing in AD might be due to an increase in activation of low frequency generators, rather than the slowing of existing sources [16]. Further research is needed to confirm these hypotheses.

Spectral and non-linear parameters that quantify the irregularity of MEG recordings also provided significant results. Statistical analyses of SE and SampEn suggest a significantly lower irregularity of MEG activity in AD patients, mainly concentrated in the centro-temporal regions. Similar spatial patterns of significant differences were observed in previous MEG studies that reported a lateralization of the reduction in entropy. Specifically, previous analyses using several spectral entropies (Shannon, Tsallis and Rényi entropy) suggested that the most significant differences between AD patients and controls were observed in both lateral cerebral regions, though the right one exhibited slightly lower $p$-values than the left one [33]. This result is related to the hemispheric asymmetry of $R P$ values and supports the notion that AD patients in our study have moderate to severe cognitive impairment. Our findings are in agreement with previous MEG studies reporting a global decrease in irregularity associated with $\mathrm{AD}$ found using several spectral entropies [22, 32] and non-linear irregularity quantifiers [26, 27, 29, 32, 34].

Regarding the non-linear parameters that quantify the complexity of MEG recordings, our findings show significantly lower $L Z C$ and $H F D$ values in AD patients than controls. Significant differences were mainly in anterior and central regions, though posterior and right lateral regions also achieved statistically significant results. Previous MEG studies also reported a loss of complexity associated with AD using several non-linear parameters [25-29, 31]. A similar pattern of abnormalities was reported in an MEG study using $H F D$, where the highest classification accuracies were 
found in the anterior, central and right lateral regions [30]. Analogous to spectral parameters, non-linear parameters exhibited a hemispheric asymmetry in the patterns of significant differences. Our HFD results showed lower $p$-values in the right lateral region than in the left lateral one, reflecting the moderate to severe cognitive deficit of the AD patients in our study.

Owing to the different natures of the parameters, the decrease in irregularity and complexity should be interpreted from two points of view. The spectral parameter (i.e., $S E$ ) suggests that $\mathrm{AD}$ is accompanied with a loss of frequency components. Thus, the spectral content of AD patients' MEG activity is more uniform than controls. The change in information entropy within the EEG may reflect a real variation in cortical functional organization [63]. Therefore, the decrease in irregularity can be associated with both loss of information content [64] and decrease in information processing at the cerebral cortex [63]. Closely related to the previous idea, the decrease in irregularity and complexity demonstrated by non-linear parameters (i.e., SampEn, LZC and HFD) could be partially explained by a decrease of dynamical complexity in some parts of the brain. Diverse mechanisms have been proposed to explain these abnormalities in the electromagnetic brain activity. The analyses with various non-linear parameters, such as $D_{2}, L 1$, neural complexity and multiscale entropy, revealed that brain dynamics in AD are characterized by a decrease in complexity of the electromagnetic brain activity [31, $65,66]$, which could be related to a degradation in the flexibility of the brain to process information [67]. Likewise, AD has been described as a disconnection syndrome, where the interactions between brain regions are strongly affected by anatomical disconnections among different cortical regions [68] or reduced cholinergic coupling interactions between cortical neurons [12]. On the basis of the previous findings, some studies hypothesized that the decrease in dynamical complexity could be due to neuronal death, a general effect of neurotransmitter deficits or loss of connectivity of local neural networks $[12,13]$. On the other hand, some studies suggested that the loss of complexity associated with AD may be frequency-dependent and may be influenced by the pattern of increases and decreases of functional connectivity [31, 41]. Certainly, further research should be conducted to assess the role of specific frequency bands in the complexity patterns usually associated with AD.

Further inspection of results shows that the $S E$ reached both the highest accuracy $(82.3 \%)$ in the anterior region and the highest AUC in the right lateral region (0.806). Similar AUCs and slightly lower accuracies were obtained by $R P$ (delta) in the anterior region (80.7\%, accuracy; $0.788, A U C)$ and $I A F$ in the central region $(80.7 \%$, accuracy; 0.803 , AUC). The $L Z C$ in the left lateral region had an accuracy and an AUC similar to the aforementioned parameters $(79.0 \%$ and 0.787 , respectively). Our results suggest that spectral parameters provide more information than nonlinear parameters in characterizing brain dynamics. There is little evidence that non-linear parameters are better suited to discriminate between AD patients and controls than spectral methods. Spectral and non-linear methods can yield complementary information useful to improve the accuracy in $\mathrm{AD}$ diagnosis $[22,32]$. It is noteworthy that both spectral and non-linear parameters achieved classification statistics favorably comparable with those reported in previous MEG studies [20, 27, 29, 32, 40]. Although the accuracies reported 
in some published works were higher than $80.7 \%$, comparisons should be established with caution considering the small sample sizes [22, 25, 27, 30, 32-34].

Some methodological and clinical findings of our study merit further consideration. None of the patients were taking any medication which could affect MEG activity during the study. Therefore, it is impossible that the abnormal slowing, irregularity and complexity patterns found in our patients could be drug-related. However, other neurodegenerative diseases, such as mild cognitive impairment and vascular dementia, etc., also elicit similar abnormal patterns in EEG and MEG activity. Further research should be conducted to compare different patterns of abnormalities associated with these disorders. It is worth mentioning that the applied statistical methodology is adequate to draw accurate statistical conclusions regarding the spatial distribution of significant differences. However, magnetic activity outside the head is mainly generated by tangential currents [10]. An acquisition sensor is most sensitive to current generators that are peripheral to the sensor area. Therefore, a source analysis could be performed to avoid an erroneous interpretation of findings derived from spatial patterns.

\section{CONCLUSION}

There is increasing evidence that $\mathrm{AD}$ is associated with a slowing of brain rhythms, together with a decrease in irregularity and complexity of the brain networks. Our findings support that AD elicits an abnormal brain dynamics. Furthermore, our results revealed that different spatial patterns of abnormalities detected by different spectral and nonlinear parameters are present in MEG activity of AD patients and control subjects. The classification statistics suggest that both spectral and non-linear parameters can be useful in discriminating between $\mathrm{AD}$ patients and healthy controls. These parameters may lead to a better understanding of the underlying brain dynamics in $\mathrm{AD}$ and can provide useful descriptors to characterize the spontaneous MEG activity in $\mathrm{AD}$.

\section{ACKNOWLEDGEMENTS}

This work was supported in part by "Ministerio de Ciencia e Innovación" under projects TEC2008-02241 and TEC2011-22987. The authors would like to thank the "Centro de Magnetoencefalografía Dr. Pérez-Modrego" of the Complutense University of Madrid for supplying the MEG recordings analyzed in this study.

\section{CONFLICT OF INTEREST}

The authors indicated no potential conflicts of interest.

\section{REFERENCES}

[1] Jalbert JJ, Daiello LA, Lapane KL. Dementia of the Alzheimer type. Epidemiologic Reviews. 2008, 30: $15-34$.

[2] Blennow K, de Leon MJ, Zetterberg H. Alzheimer's disease. Lancet. 2006, 368(9533): 387-403.

[3] Kalaria RN, Maestre GE Arizaga R, Friedland RP, Galasko D, Hall K, Luchsinger JA, Ogunniyi A, Perry EK, Potocnik F, Prince M, Stewart R, Wimo A, Zhang ZX, Antuono P, World Federation of Neurology Dementia Research Group. Alzheimer's disease and vascular dementia in developing countries: prevalence, management, and risk factors. Lancet Neurology. 2008, 7(9): 812-826. 
[4] McKhann G, Drachman D, Folstein M, Katzman R, Price D, Stadlan EM. Clinical diagnosis of Alzheimer's disease: report of NINCDS-ADRDA work group under the auspices of department of health and human services task force on Alzheimer's disease. Neurology. 1984, 34(7): 939-944.

[5] Knopman DS, DeKosky ST, Cummings JL, Chui H, Corey-Bloom J, Relkin N, Small GW, Miller B, Stevens JC. Practice parameter: Diagnosis of dementia (an evidence-based review). Report of the Quality Standards Subcommittee of the American Academy of Neurology. Neurology. 2001, 56(9): 1143-1153.

[6] Dubois B, Feldman HH, Jacova C, Dekosky ST, Barberger-Gateau P, Cummings J, Delacourte A, Galasko D, Gauthier S, Jicha G, Meguro K, O’brien J, Pasquier F, Robert P, Rossor M, Salloway S, Stern Y, Visser PJ, Scheltens P. Research criteria for the diagnosis of Alzheimer's disease: revising the NINCDS-ADRDA criteria. Lancet Neurology. 2007, 6(8): 734-746.

[7] Shineman DW, Fillit HM. Novel strategies for the prevention of dementia from Alzheimer's disease. Dialogues in Clinical Neuroscience. 2009, 11(2): 129-134.

[8] Cummings JL. Alzheimer's disease. The New England Journal of Medicine. 2004, 351(1): 56-67.

[9] Rossini PM, Rossi S, Babiloni C, Polich J. Clinical neurophysiology of aging brain: From normal aging to neurodegeneration. Progress in Neurobiology. 2007, 83(6): 375-400.

[10] Hari R, Magnetoencephalography in clinical neurophysiological assessment of human cortical functions, in: Ernst Niedermeyer, Fernando Lopes da Silva (eds). Electroencephalography: Basic Principles, Clinical Applications, and Related Fields, 5th ed., Lippincontt Williams \& Wilkins, Philadelphia, USA, 2005, 1165-1197.

[11] Nunez PL, Wingeier BM, Silberstein RB. Spatial-Temporal structures of human alpha rhythms: Microcurrents sources, multiscale elements, and global binding of local networks. Human Brain Mapping. 2001, 13(3): 125-164.

[12] Jeong J. EEG dynamics in patients with Alzheimer's disease. Clinical Neurophysiology. 2004, 115(7): 1490-1505.

[13] Stam CJ. Nonlinear dynamical analysis of EEG and MEG: Review of an emerging field. Clinical Neurophysiology. 2005, 116(10): 2266-2301.

[14] Stam CJ. Use of magnetoencephalography (MEG) to study functional brain networks in neurodegenerative disorders. Journal of the Neurological Sciences. 2010, 289(1-2): 128-134.

[15] Berendse HW, Verbunt JPA, Scheltens Ph, van Dijk BW, Jonkman EJ. Magnetoencephalographic analysis of cortical activity in Alzheimer's disease: a pilot study. Clinical Neurophysiology. 2000, 111(4): 604-612.

[16] Osipova D, Ahveninen J, Jensen O, Ylikoski A, Pekkonen E. Altered generation of spontaneous oscillations in Alzheimer's disease. NeuroImage. 2005, 27(4): 835-841.

[17] Fernández A, Hornero R, Mayo A, Poza J, Gil-Gregorio P, Ortiz T. MEG spectral profile in Alzheimer's disease and mild cognitive impairment. Clinical Neurophysiology. 2006, 117(2): 306-314.

[18] Fernández A, Hornero R, Mayo A, Poza J, Maestú F, Ortiz T. Quantitative magnetoencephalography of spontaneous brain activity in Alzheimer disease: an exhaustive frequency analysis. Alzheimer Disease and Associated Disorders. 2006, 20(3): 153-159.

[19] de Haan W, Stam CJ, Jones BF, Zuiderwijk IM, van Dijk BW, Scheltens P. Resting-state oscillatory brain dynamics in Alzheimer disease. Journal of Clinical Neurophysiology. 2008, 25(4): 187-193.

[20] Poza J, Hornero R, Abásolo D, Fernández A, Mayo A. Evaluation of spectral ratio measures from spontaneous MEG recordings in patients with Alzheimer's disease. Computer Methods and Programs in Biomedicine. 2008, 90(2): 137-147.

[21] Hindriks R, Bijma F, van Dijk BW, van der Werf YD, van Someren EJ, van der Vaart AW. Dynamics underlying spontaneous human alpha oscillations: a data-driven approach. NeuroImage. 2011, 57(2): 440-451.

[22] Poza J, Hornero R, Abásolo D, Fernández A, García M. Extraction of spectral based measures from MEG background oscillations in Alzheimer's disease. Medical Engineering and Physics. 2007, 29(10): 1073-1083. 
[23] Osipova D, Rantanen K, Ahveninen J, Ylikoski R, Häppölä O, Strandberg T, Pekkonen E. Source estimation of spontaneous MEG oscillations in mild cognitive impairment. Neuroscience Letters. 2006, 405(1-2): 57-61.

[24] Abatzoglou I, Anninos P, Adamopoulos A, Koukourakis M. Nonlinear analysis of brain magnetoencephalographic activity in Alzheimer disease patients. Acta Neurologica Belgica. 2007, 107(2): 34-39.

[25] Gómez C, Hornero R, Abásolo D, Fernández A, López M. Complexity analysis of the magnetoencephalogram background activity in Alzheimer's disease patients. Medical Engineering and Physics. 2006, 28(9): 851-859.

[26] Gómez C, Hornero R, Abásolo D, Fernández A, Escudero J. Analysis of MEG background activity in Alzheimer's disease using non-linear methods and ANFIS. Annals of Biomedical Engineering. 2009, 37(3): 586-594.

[27] Hornero R, Abásolo D, Escudero J, Gómez C. Nonlinear analysis of electroencephalogram and magnetoencephalogram recordings in patients with Alzheimer's disease. Philosophical Transactions of the Royal Society A, Mathematical, Physical, and Engineering Sciences. 2009, 367(1887): 317-336.

[28] Fernández A, Hornero R, Gómez C, Turrero A, Gil-Gregorio P, Matías-Santos J, Ortiz T. Complexity analysis of spontaneous brain activity in Alzheimer's Disease and Mild Cognitive Impairment. Alzheimer Disease and Associated Disorders. 2010, 24(1): 1-10.

[29] Gómez C, Hornero R. Entropy and complexity analyses in Alzheimer's disease: an MEG study. The Open Biomedical Engineering Journal. 2010, 4: 223-235.

[30] Gómez C, Mediavilla A, Hornero R, Abásolo D, Fernández A. Use of the Higuchi’s fractal dimension for the analysis of MEG recordings from Alzheimer's disease patients. Medical Engineering and Physics. 2009, 31(3): 306-313.

[31] van Cappellen van Walsum A-M, Pijnenburg YAL, Berendse HW, van Dijk BW, Knol DL, Scheltens P, Stam CJ. A neural complexity measure applied to MEG data in Alzheimer's disease. Clinical Neurophysiology. 2003, 114(6): 1034-1040.

[32] Hornero R, Escudero J, Fernández A, Poza J, Gómez C. Spectral and Non-linear Analyses of MEG Background Activity in Patients with Alzheimer's Disease. IEEE Transactions on Biomedical Engineering. 2008, 55(6): 1658-1665.

[33] Poza J, Escudero J, Hornero R, Fernández A, Sanchez CI. Regional analysis of spontaneous MEG rhythms in patients with Alzheimer's disease using spectral entropies. Annals of Biomedical Engineering. 2008, 36(1): 141-152.

[34] Gómez C, Hornero R, Abásolo D, Fernández A, Escudero J. Analysis of the magnetoencephalogram background activity in Alzheimer's disease patients with auto mutual information. Computers Methods and Programs in Biomedicine. 2007, 87(3): 239-247.

[35] Stam CJ, van Cappellen van Walsum AM, Pijnenburg YA, Berendse HW, de Munck JC, Scheltens P, van Dijk BW. Generalized synchronization of MEG recordings in Alzheimer's Disease: evidence for involvement of the gamma band. Journal of Clinical Neurophysiology. 2002, 19(6): 562-574.

[36] Franciotti R, Iacono D, Della Penna S, Pizzella V, Torquati K, Onofrj M, Romani GL. Cortical rhythms reactivity in AD, LBD and normal subjects. A quantitative MEG study. Neurobiology of Aging. 2006, 27(8): 1100-1109.

[37] Montez T, Poil SS, Jones BF, Manshanden I, Verbunt JP, van Dijk BW, Brussaard AB, van Ooyen A, Stam CJ, Scheltens P, Linkenkaer-Hansen K. Altered temporal correlations in parietal alpha and prefrontal theta oscillations in early-stage Alzheimer disease. Proceedings of the National Academy of Sciences of the United States of America. 2009, 106(5): 1614-1619.

[38] Gómez C, Stam CJ, Hornero R, Fernández A, Maestú F. Disturbed beta band functional connectivity in patients with mild cognitive impairment: An MEG study. IEEE Transactions on Biomedical Engineering. 2009, 56(6): 1683-1690.

[39] Stam CJ, de Haan W, Daffertshofer A, Jones BF, Manshanden I, van Cappellen van Walsum AM, Montez T, Verbunt JP, de Munck JC, van Dijk BW, Berendse HW, Scheltens P. Graph theoretical 
analysis of magnetoencephalographic functional connectivity in Alzheimer's disease. Brain. 2009, 132(Pt 1): 213-224.

[40] Alonso JF, Poza J, Mañanas MA, Romero S, Fernández A, Hornero R. MEG connectivity analysis in patients with Alzheimer's disease using cross mutual information and spectral coherence. Annals of Biomedical Engineering. 2010, 39(1): 524-536.

[41] Stam CJ, Jones BF, Manshanden I, van Cappellen van Walsum AM, Montez T, Verbunt JP, de Munck JC, van Dijk BW, Berendse HW, Scheltens P. Magnetoencephalographic evaluation of resting-state functional connectivity in Alzheimer's disease. NeuroImage. 2006, 32(3): 1335-1344.

[42] Auer S, Reisberg B. The GDS/FAST staging system. International Psychogeriatrics. 1997, 9(1): 167-171.

[43] Rodriguez G, Copello F, Vitali P, Perego G, Nobili F. EEG spectral profile to stage Alzheimer's disease. Clinical Neurophysiology. 1999, 110(10): 1831-1837.

[44] Klimesch W. EEG alpha and theta oscillations reflect cognitive and memory performance: a review analysis. Brain Research Review. 1999, 29(2-3): 169-195.

[45] Powell GE, Percival IC. A spectral entropy method for distinguishing regular and irregular motion of Hamiltonian systems. Journal of Physics A: Mathematical and General. 1979, 12(11): 2053-2071.

[46] Eckmann JP, Ruelle D. Fundamental limitations for estimating dimensions and Lyapunov exponents in dynamical systems. Physica D: Nonlinear Phenomena. 1992, 56(2-3): 185-187.

[47] Richman JS, Moorman JR. Physiological time-series analysis using approximate entropy and sample entropy. American Journal of Physiology. Heart and Circulatory Physiology. 2000, 278(6): H2039H2049.

[48] Lempel A, Ziv J. On the complexity of finite sequences. IEEE Transactions on Information Theory. 1976, 22(1): 75-81.

[49] Zhang XS, Roy RJ, Jensen EW. EEG complexity as a measure of depth of anesthesia for patients. IEEE Transactions on Biomedical Engineering. 2001, 48(12): 1424-1433.

[50] Nagarajan R. Quantifying physiological data with Lempel-Ziv complexity - certain issues. IEEE Transactions on Biomedical Engineering. 2002, 49(11): 1371-1373.

[51] Mandelbrot B. Fractals: Form, Chance, and Dimension, Freeman, San Francisco, 1977.

[52] Accardo A, Affinito M, Carrozzi M, Bouquet F. Use of the fractal dimension for the analysis of electroencephalographic time series. Biological Cybernetics. 1997, 77(5): 339-350.

[53] Maragos P, Sun FK. Measuring the fractal dimension of signals: morphological covers and iterative optimization. IEEE Transactions on Signal Processing. 1983, 41(1), 108-121.

[54] Katz M. Fractals and the analysis of waveforms. Computers in Biology and Medicine. 1988, 18(3): $145-156$.

[55] Higuchi T. Approach to an irregular time series on the basis of the fractal theory. Physica D: Nonlinear Phenomena. 1988, 31(2): 277-283.

[56] Sleigh JW, Steyn-Ross DA, Grant C, Ludbrook G. Cortical entropy changes with general anaesthesia: theory and experiment. Physiological Measurement. 2004, 25(4): 921-934.

[57] Bendat J, Piersol A. Random Data Analysis and Measurement Procedures, Wiley, New York, 2000.

[58] Fernández A, Maestú F, Amo C, Gil P, Fehr T, Wienbruch C, Rockstroh B, Elbert T, Ortiz T. Focal temporoparietal slow activity in Alzheimer's disease revealed by magnetoencephalography. Biological Psychiatry. 2002, 52(7). 764-770.

[59] Fernández A, Turrero A, Zuluaga P, Gil P, Maestú F, Campo P, Ortiz T. Magnetoencephalographic parietal delta dipole density in mild cognitive impairment: preliminary results of a method to estimate the risk of developing Alzheimer disease. Archives of Neurology. 2006, 63(3). 427-430.

[60] Giannakopoulos P, Kövari E, Herrmann FR, Hof PR, Bouras C. Interhemispheric distribution of Alzheimer disease and vascular pathology in brain aging. Stroke. 2009, 40(3): 983-986.

[61] Huang C, Wahlund LO, Dierks T, Julin P, Winblad B, Jelic V. Discrimination of Alzheimer's disease and mild cognitive impairment by equivalent EEG sources: a cross-sectional and longitudinal study. Clinical Neurophysiology. 2000, 111(11): 1961-1967. 
[62] Osipova D, Ahveninen J, Kaakkola S, Jääskeläinen IP, Huttunen J, Pekkonen E. Effects of scopolamine on MEG spectral power and coherence in elderly subjects. Clinical Neurophysiology. 2003, 114(10): 1902-1907.

[63] Kannathal N, Choob ML, Acharyab UR, Sadasivana PK. Entropies for detection of epilepsy in EEG. Computer Methods and Programs in Biomedicine. 2005, 80(3): 187-194.

[64] Baraniuk RG, Flandrin P, Janssen AJEM, Michel OJJ. Measuring time-frequency information content using the Rényi entropies. IEEE Transactions on Information Theory. 2001, 47(4): 1391-1409.

[65] Besthorn C, Sattel H, Geiger-Kabisch C, Zerfass R, Förstl H. Parameters of EEG dimensional complexity in Alzheimer's disease. Electroencephalography and Clinical Neurophysiology. 1995, 95(2): 84-89.

[66] Escudero J, Abásolo D, Hornero R, Espino P, López M. Analysis of electroencephalograms in Alzheimer's disease patients with multiscale entropy. Physiological Measurement. 2006, 27(11): 1091-1106.

[67] Jeong J, Kim SJ, Han SH. Non-linear dynamical analysis of the EEG in Alzheimer's disease with optimal embedding dimension. Electroencephalography and Clinical Neurophysiology. 1998, 106(3): $220-228$.

[68] Jelles B, van Birgelen JH, Slaets JPJ, Hekster REM, Jonkman EJ, Stam CJ. Decrease of non-linear structure in the EEG of Alzheimer patients compared to healthy controls. Clinical Neurophysiology. 1999, 110(7): 1159-1167. 



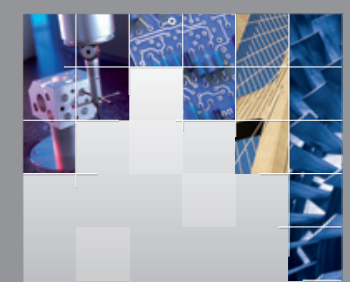

\section{Enfincering}
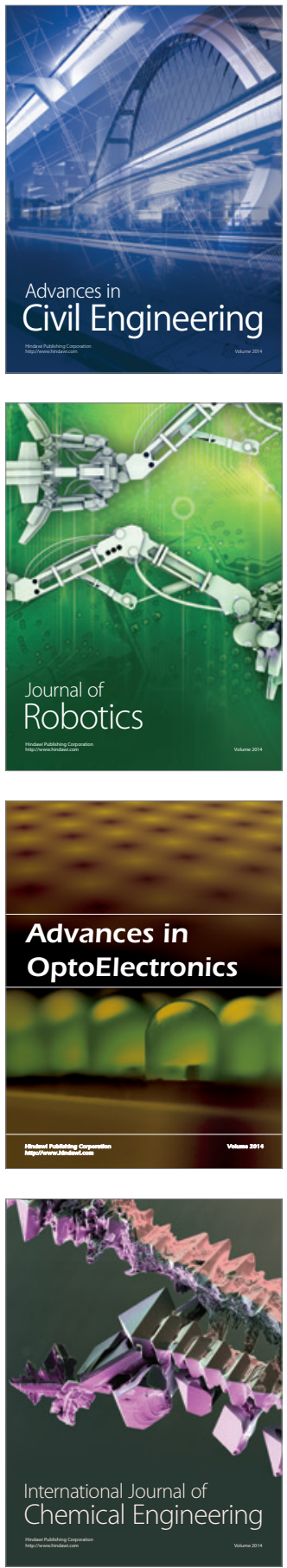

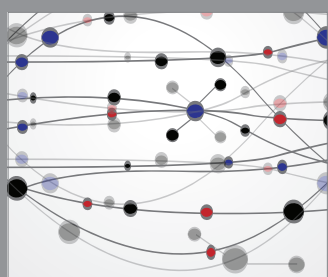

The Scientific World Journal

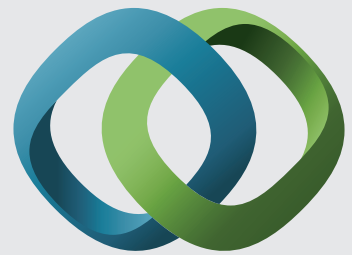

\section{Hindawi}

Submit your manuscripts at

http://www.hindawi.com
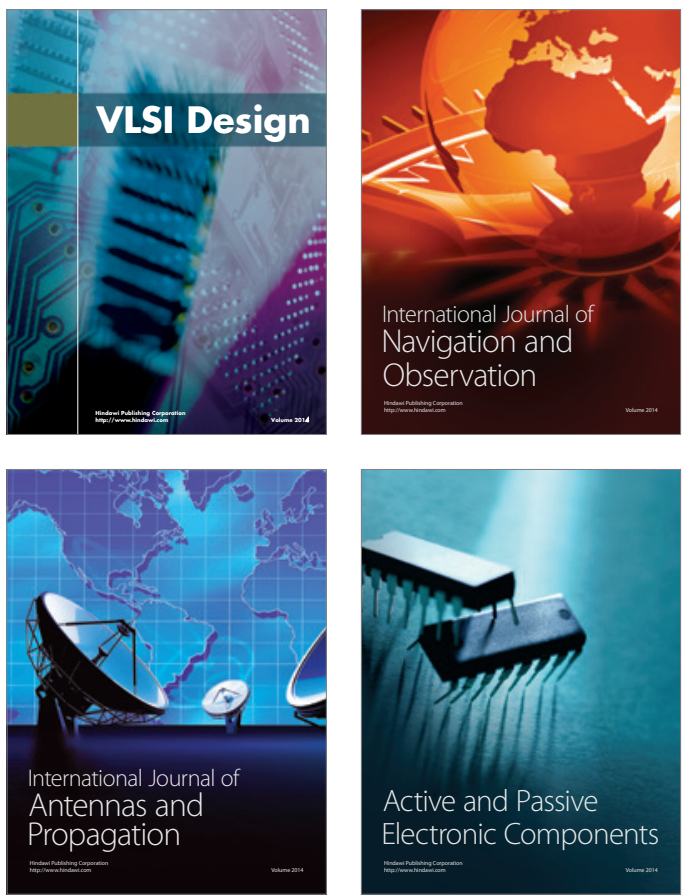
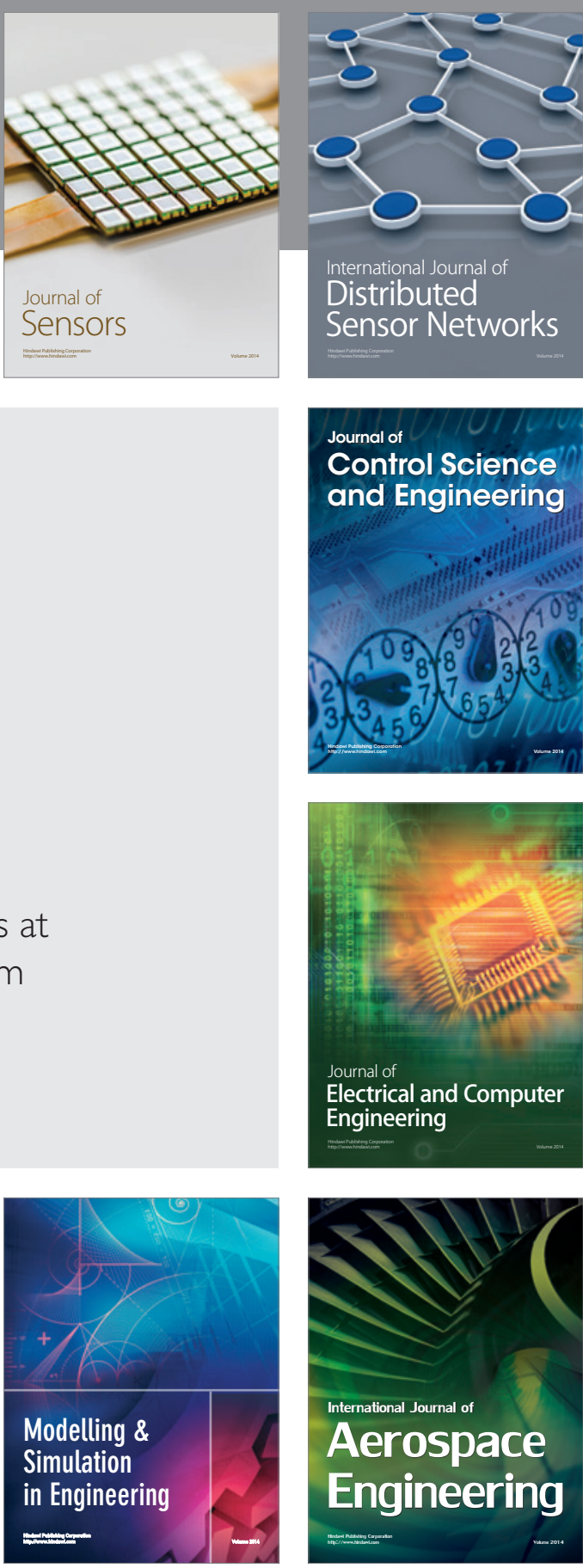

International Journal of

Distributed

Sensor Networks

Journal of

Control Science

and Engineering
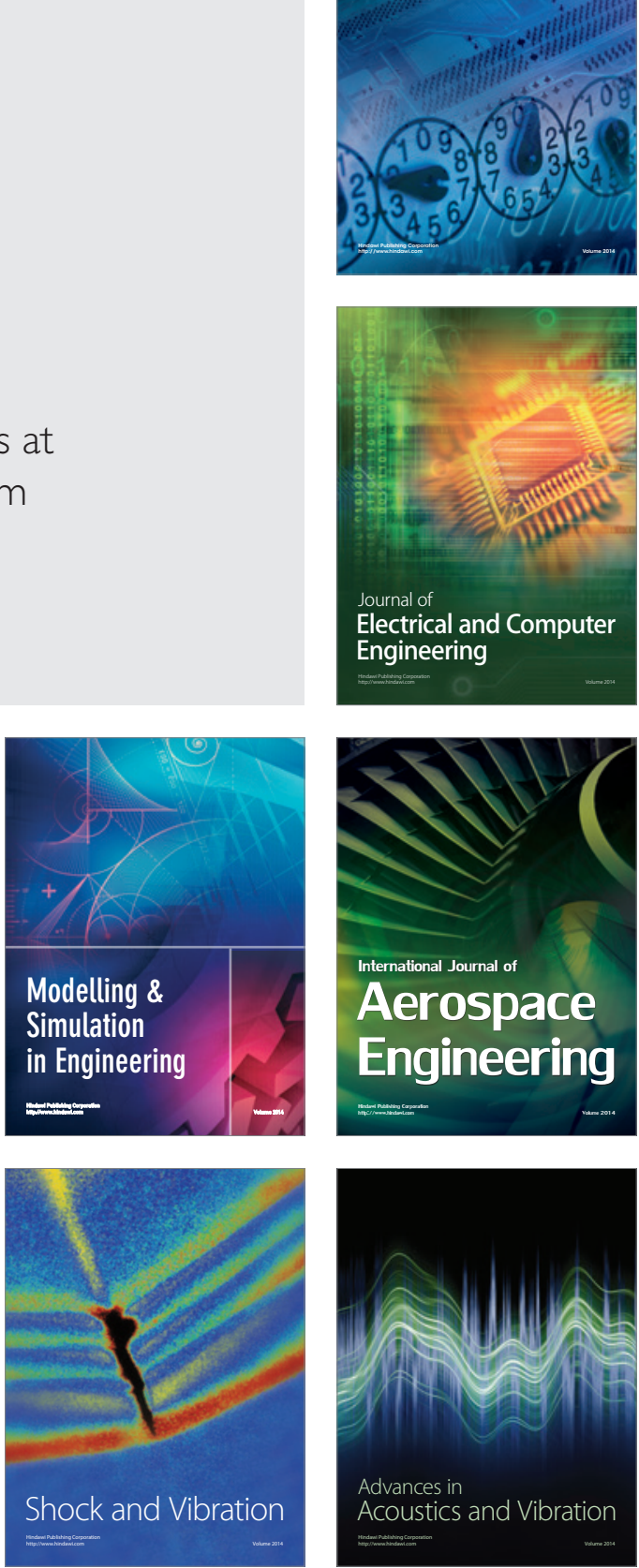